\title{
Spectroscopy: a versatile sensing tool for cost-effective and rapid detection of novel coronavirus (COVID-19)
}

\author{
Ujjal Kumar Sur ${ }^{1} \cdot$ Chittaranjan Santra $^{2}$
}

Received: 25 October 2021 / Accepted: 10 February 2022 / Published online: 28 February 2022

(c) Qatar University and Springer Nature Switzerland AG 2022

\begin{abstract}
The deadly novel coronavirus SARS-CoV-2 is responsible for COVID-19, which was first recognized in Wuhan, China, in December 2019. Rapid identification at primary stage of the novel coronavirus, SARS-CoV-2, is important to restrict it and prevent the pandemic. Real-time RT-PCR assays are the best diagnostic tests presently available for SARS-CoV-2 detection, which are highly sensitive, even though expensive equipment and trained technicians are necessary. Furthermore, the method has moderately long time bound. This deadly viral infection can also be detected by applying various spectroscopic techniques as spectroscopy can provide fast, precise identification and monitoring, leading to the overall understanding of its mutation rates, which will further facilitate antiviral drug development as well as vaccine development. It is an innovative and non-invasive technique for combating the spread of novel coronavirus. This review article demonstrates the application of various spectroscopic techniques to detect COVID-19 rapidly. Different spectroscopy-based detection protocols and additional development of new, novel sensors and biosensors along with diagnostic kits had been described here stressing the status of sensitive diagnostic systems to handle with the COVID-19 outbreak.
\end{abstract}

Keywords COVID-19 - Spectroscopy $\cdot$ Diagnostics $\cdot$ RT-PCR $\cdot$ Raman spectroscopy $\cdot$ Vibrational spectroscopy $\cdot$ Surfaceenhanced Raman scattering

\section{Introduction}

Coronavirus, which is the main cause of common cold, was detected for the first time during 1960s. Coronavirus is an enveloped positive-sense single-stranded RNA having a pleomorphic or spherical shape with projections of spike glycoproteins. Different subtype variants such as alpha, beta, gamma, and delta coronavirus are existing together along with serotype of each subtype. The human coronavirus (OC43-like and 229E-like) can also exist further in other living organisms such as bats, pangolins, pigs, birds, cats, dogs, and mice. This virus will spread through aerial droplets of sneezing and coughing and the virus will replicate in the ciliated epithelium causing harm to living cells and inflammatory reactions at the infected location $[1,2]$. The

Ujjal Kumar Sur

uksur99@yahoo.co.in

1 Department of Chemistry, Behala College, University of Calcutta, Parnashree, Kolkata 700060, West Bengal, India

2 Department of Chemistry (Ex), Netaji Nagar Day College, Kolkata 700092, India club-shaped protein spikes on the surface of virus, which can be monitored under a transmission electron microscope (TEM) [3, 4], have given rise the name "coronavirus." The dimension of this deadly virus is between 50 and $100 \mathrm{~nm}$ as observed by TEM study [3, 4]. Figure 1 shows the TEM image along with the schematic picture of the COVID-19 virus. SARS-CoV-2 is a RNA virus from the Coronaviridae family, which is covered by an envelope with protein spikes having four structural proteins such as spike surface glycoprotein (S), small envelope protein (E), matrix membrane glycoprotein $(\mathrm{M})$, and nucleocapsid protein $(\mathrm{N})$ as illustrated in Fig. 1.

It has identified angiotensin receptor 2 (ACE2) as the receptor through which the virus enters the respiratory mucosa. Mechanisms of coronavirus cell entry is mediated by the viral spike protein with ACE2 receptor.

The severe acute respiratory syndrome (SARS), the Middle East respiratory syndrome (MERS), and the 2019 outbreak of COVID-19 worldwide pandemic [5] are all caused by the beta class of coronavirus showing symptoms of dry cough, fatigue, and breathing problems as initial symptoms of a SARS-CoV-2-infected patients. This virus is much more

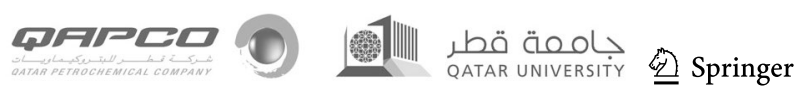


Fig. 1 TEM image (Reprinted with permission from Goldsmith et al. [3], Copyright (2004) Centers for Disease Control and Prevention, USA) and schematic picture of COVID-19 virus

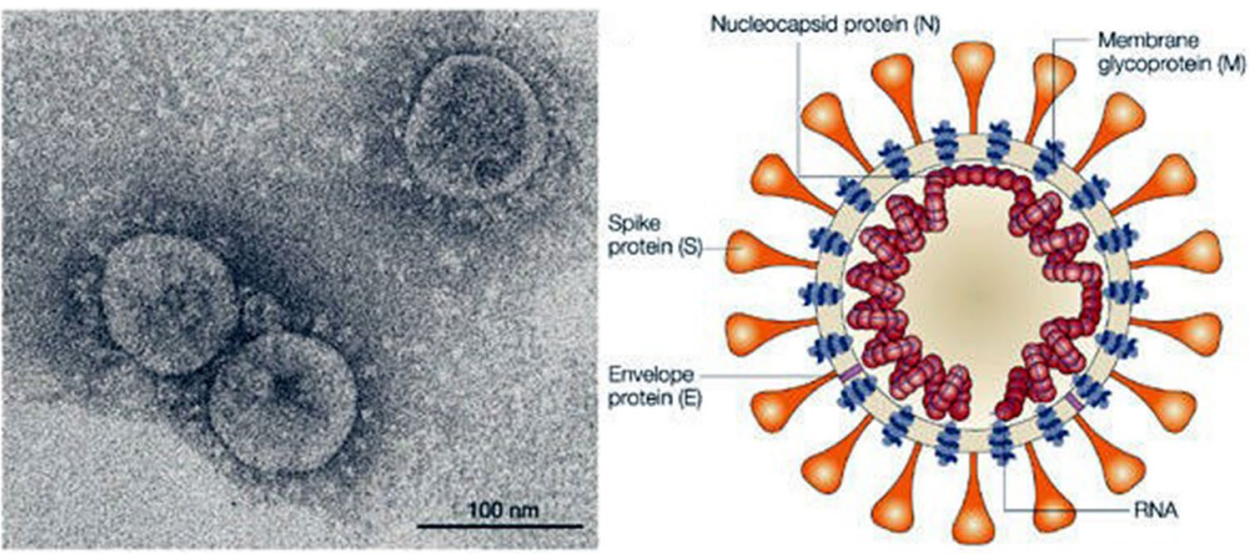

infectious and affects the lower respiratory system (larynx, trachea, bronchi, and lungs) that causes viral pneumonia and also attacks the human heart, liver, kidneys, and gastrointestinal tract, and the central nervous system can also be affected, causing patients to experience more severe multiorgan dysfunctions.

Subsequently, the observed COVID-19 fatality rate is higher for older group people having age above 60 years making them the most vulnerable to this deadly virus [2]. Nasal congestion, sore throat, breathing difficulties, chest pains, chills, kidney failure, and pneumonia are some of the symptoms of this deadly virus.

COVID-19 was detected for the first time in December 31, 2019, in Wuhan, China, when China informed the World Health Organization (WHO), and the whole world experienced COVID-19 for the first time [6-9]. Until now, no specific drugs are accessible commercially for the diagnosis of COVID-19. Therefore, a rapid and accurate diagnosis for SARS-CoV-2, followed by self-isolation, is vital to slow down the spread of COVID-19 and for effective execution of control and suppression approaches.

The current global epidemic of this evolving coronavirus is growing terrifyingly and existing means of diagnosis such as reverse transcriptase polymerase chain reaction (RT-PCR) are not ideal for cost-effectiveness, accuracy, and time duration of detection of this deadly virus [8]. Consequently, a quicker, cheaper, and simpler method for the detection of viral infections in biofluids like saliva or blood is essential towards controlling and prevention of the proliferation of this deadly disease.

Spectroscopy has novel applications in medical and biomedical research, as both Raman and infrared (IR) spectroscopies can be applied in the diagnosis of viral and bacterial infections with circumspection $[10,11]$ by providing early detection, diagnosis, and monitoring of several human diseases. Bacteria and viruses can be studied in details with the help of recent technologies such as nanotechnology and biotechnology, which are capable of providing immediate and rapid information about the overall composition of biological samples and species [12]. Novel spectroscopic analytical techniques such as proton nuclear magnetic resonance, Raman spectroscopy, and IR spectroscopy can be employed to investigate various complex biological clinical samples such as saliva, urine, blood, breast milk, CVF (cervical vaginal fluid), stool, and sputum, with extraordinary high efficiency and resolution [13, 14]. All these analytical techniques are vibrational spectroscopic techniques which can provide data about the molecular vibrations of the chemical structures of molecules with the detection of the viral proteins or antibody proteins from the immune system response. The vibrational spectrum obtained from both IR and Raman spectroscopic techniques can be analyzed and further utilized for the detection of glycoproteins and nucleic acids of viruses providing the means for the quick and accurate diagnosis and continuous monitoring [15-17].

Vibrational spectroscopy will assist in the overall understanding of infection process of this contagious disease by quick, precise detection and monitoring, leading to acquire information about viral mutation and drug development, and also for development of vaccines for COVID-19.

Although a large number of review articles had been written on the various aspects of COVID-19 [8, 9, 18, 19], only a few articles cover the diagnostic aspects and detection methods of this deadly virus [20-24]. There are few papers available in the literature which had shown the use of spectroscopic techniques for the detection of this deadly virus [25-28]. There is also no review article written on the demonstration of utilization of different spectroscopic techniques for the detection of COVID-19 viral infections as per our knowledge. The main current targets of SARS-CoV-2 sequence detection include three conserved gene sequences in the viral genome, which include the open reading frame (ORF), N gene, and E gene [18, 19]. The specimens for testing can be nasopharyngeal swabs, sputum, other lower respiratory tract secretions, blood, and faeces. The objective of this review article is to provide updated overview

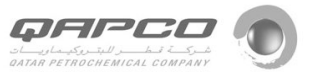


of application of spectroscopy as the diagnostic tool for the rapid, cost-effective detection of SARS-CoV-2 describing the versatility in terms of the advantages over RT-PCR method. This review article has described the role of vibrational spectroscopy both IR and Raman for the detection of COVID-19. In addition, we have also demonstrated new and novel spectroscopic technique based on nanotechnology to detect this deadly virus. A comprehensive review of all current research on this important subject is not possible due to page limitations. Nevertheless, we only summarize a few representative examples.

\section{Detection of COVID-19 by RT-PCR method}

RT-PCR is considered to be the most conventional diagnostic test for SARS-CoV-2 [29, 30]. In this method, viral RNA is first purified from clinical samples collected from nasopharyngeal swabs, copied into DNA, and finally amplified in a PCR reaction to detect sufficient quantity of virus DNA [30]. In the laboratory, isolation of viral RNA, the RT-PCR reaction, and analysis can be automatically processed so that more than 100 samples can be simultaneously examined on a single PCR machine with a turnaround time of a few hours. Human antibody and viral antigen tests are the other monitoring tests currently available to detect COVID-19 infection [30] along with the conventional RT-PCR method. Antibodies are generated on most infected persons arising out from the response of immune system towards most pathogens and these can be detected with the help of synthetic peptides intended to imitate definite percentages of a pathogen's surface which has been exposed to the immune system. These antibody tests are carried out to detect SARS-CoV-1 and MERS-CoV in the serum of a person infected before and are effective over a wide spectrum of time duration ranging from weeks to months to years afterwards the infection and are dependent on the immunity to a given pathogen. These antibody tests are helpful to investigate the transmission of a disease and can deliver perception into the measure of an epidemic. The test can also be effective to monitor the asymptotic patients, who are responsible for the unidentified transmission chain during a viral outbreak. The antigen test works likewise excluding that antibodies produced against a pathogen are directly employed to detect this pathogen in the serum or other body fluids of infected patients. The rapid antigen test (RAT) is less sensitive than the RT-PCR test and can be commonly employed some days after a patient becomes symptomatic. The antigen test is usually less expensive than RT-PCR, and has a fast turnaround time.

Although RT-PCR is most commonly employed to detect COVID-19, it is both laborious and tedious owing to collection (especially for kids) and preparation of sample prior to the analysis. It is also identified as quantitative
PCR or qPCR and in that respect, the enhancement of DNA can be detected in real time during the progress of PCR by employing a fluorescent reporter [29, 30]. PCR testing has high sensitivity with 10 copies per reaction [30]. However, false-negative tests are responsible for the failure of detection of COVID-19-infected patients. Therefore, the accurate protocol for PCR is essential to carry out detection of COVID-19-infected patients. The commencement and development of other molecular techniques in addition to RT-PCR for the rapid and accurate detection of this deadly virus is essential for the easy and quick prevention and diagnosis of the COVID-19 global epidemic.

\section{Development of diagnostic kit for the detection of COVID-19}

Different countries had developed nucleic acid assay-based diagnostic kits to detect COVID-19 infections [31, 32].

Two single-step quantitative RT-PCR assays for $\mathrm{N}$ gene and ORF1b of sarbecovirus subgenus were developed by the researchers of University of Hong Kong. Coronavirus gene detection kit was developed by Amoy Diagnostics of Xiamen, China, while Altona Diagnostics of Hamburg, Germany, developed RT-PCR assay for the detection of Coronavirus RNA from respiratory samples. BGI group of Beijing, China, employed real-time fluorescent RT-PCR kit and metagenomic sequencing kit to monitor the mutations of coronavirus and designed nucleic acid detection kit using combinatorial probe anchor synthesis method. Table 1 illustrates the various detection kits used for the diagnosis of COVID-19 along with their advantages and drawbacks [31-33]. Recently, a cost-effective COVID-19 testing kit DiAGSure Ncov-19 was developed and designed by researchers in West Bengal, India. The cost of this novel diagnostic kit is approximately Rs 500 $(\sim$ \$) with almost $100 \%$ accuracy in detecting the virus within a brief duration of $90 \mathrm{~min}$. This indigenous kit was developed by GCC Biotech limited, a company based in India and it can provide diagnostic test up to 160 patients. The test kit comprises of a one-step qRT PCR Master Mix primer probe along with a RNA template, locally developed by this Kolkata-based biotechnological company.

Recently, scientists from IGIB, New Delhi, India, developed novel diagnostic kit Feluda CRISPR, which can detect the presence of coronavirus faster than rRTPCR test.

A Singapore-based company Breathonix, a spin-off from National University of Singapore, has developed a real-time COVID-19 breath test analyzer to detect COVID-19 within 60 s with $90 \%$ accuracy and $93 \%$ sensitivity.

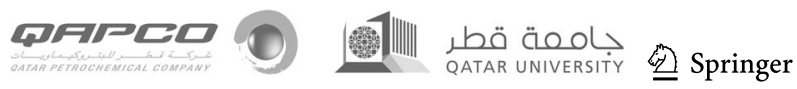


Table 1 The various detection kits used for detection of COVID-19 along with advantages and drawbacks

\begin{tabular}{|c|c|c|c|}
\hline Diagnostic test & Developer & Advantage & Drawback \\
\hline $\begin{array}{l}\text { Real time reverse transcriptase } \\
\text { PCR assays }\end{array}$ & University of Hong Kong & High sensitivity and specificity & $\begin{array}{l}\text { Not useful for detection COVID-19 } \\
\text { cases with high viral loads }\end{array}$ \\
\hline Coronavirus gene detection kit & $\begin{array}{l}\text { Amoy Diagnostics (Xiamen, } \\
\text { China) }\end{array}$ & $\begin{array}{l}99.8 \% \text { accuracy with limit of } \\
\text { detection } 500 \text { copies } / \mathrm{mL}\end{array}$ & Low sensitivity \\
\hline Nucleic acid assay & $\begin{array}{l}\text { Chinese national institute for viral } \\
\text { disease control and prevention }\end{array}$ & $\begin{array}{l}\text { Comparable sensitivity and speci- } \\
\text { ficity to commercial qRT-PCR } \\
\text { kits in terms of operation and } \\
\text { turnaround time }\end{array}$ & Moderate sensitivity \\
\hline Real-time PCR assay & $\begin{array}{l}\text { Altona Diagnostics (Hamburg, } \\
\text { Germany) }\end{array}$ & $\begin{array}{l}\text { Comparable with various real- } \\
\text { time PCR platforms }\end{array}$ & $\begin{array}{l}\text { For research use only, not for use in } \\
\text { diagnostic procedures }\end{array}$ \\
\hline $\begin{array}{l}\text { Real-time fluorescent } \\
\text { RT-PCR kit }\end{array}$ & BGI Group (Beijing) & $\begin{array}{l}\text { Highly sensitive with limit of } \\
\text { detection } 100 \text { copies/mL. Highly } \\
\text { specific with no cross-reactivity } \\
\text { with } 54 \text { human respiratory } \\
\text { pathogens. High throughput }\end{array}$ & $\begin{array}{l}\text { Stored at a temperature } \\
\text { below }-20{ }^{\circ} \mathrm{C}\end{array}$ \\
\hline Nucleic acid detection kit & BGI Group & High sensitivity, wide linear range & $\begin{array}{l}\text { Did not cross-react with other } \\
\text { circulating coronaviruses }\end{array}$ \\
\hline COVID-19 Go-Strips & Biomeme & $\begin{array}{l}\text { Portable on-site testing with high } \\
\text { sensitivity }\end{array}$ & For in vitro diagnostic use only \\
\hline TaqMan 2019-nCoV Assay Kit & Thermo Fisher Scientific & $\begin{array}{l}\text { High-throughput, low cost, high- } \\
\text { sensitive commercial diagnostic } \\
\text { kit }\end{array}$ & $\begin{array}{l}\text { Stored at a temperature } \\
\text { below }-20{ }^{\circ} \mathrm{C}\end{array}$ \\
\hline $\begin{array}{l}\text { DiAGSure Ncov-19 detection } \\
\text { assay }\end{array}$ & $\begin{array}{l}\text { GCC Biotech limited, Kolkata, } \\
\text { India }\end{array}$ & $\begin{array}{l}\text { Low-priced COVID-19 testing kit } \\
\text { with nearly } 100 \% \text { accuracy in a } \\
\text { short span of } 90 \mathrm{~min}\end{array}$ & $\begin{array}{l}\text { It cannot provide information on } \\
\text { other diseases or infection }\end{array}$ \\
\hline Feluda CRISPR test kit & IGIB, New Delhi, India & $\begin{array}{l}\text { The test kit had } 96 \% \text { sensitivity } \\
\text { and } 98 \% \text { specificity }\end{array}$ & $\begin{array}{l}\text { The virus may only be detectable in } \\
\text { sputum or nasopharyngeal swab } \\
\text { but not necessarily at both loca- } \\
\text { tions at the same time }\end{array}$ \\
\hline $\begin{array}{l}\text { Real-time COVID-19 breath test } \\
\text { analyzer }\end{array}$ & Breathonix, Singapore & $\begin{array}{l}\text { Real-time COVID-19 breath test } \\
\text { analyzer to detect COVID-19 } \\
\text { within } 60 \mathrm{~s} \text { with } 90 \% \text { accuracy } \\
\text { and } 93 \% \text { sensitivity }\end{array}$ & $\begin{array}{l}\text { Not useful for detection COVID-19 } \\
\text { cases with low viral loads }\end{array}$ \\
\hline
\end{tabular}

\section{Detection of coronavirus using mass spectrometry}

Mass spectrometry (MS) can be utilized to obtain diagnostic data and provide genomic information about SARS-CoV-2 virus. A simple MS-based diagnostic protocol was developed by Ihling et al. [34] to explicitly detect SARS-CoV-2 proteins from gargle solution samples of COVID-19-infected patients. In this diagnostic protocol, precipitation of acetone and tryptic digestion of protein within the gargle solution were carried out followed by a targeted MS analysis. This MS-based technique can recognize peptides initiating from SARS-CoV-2 virus nucleoprotein exclusively. It can be applied on bronchoalveolar lavage and will provide sensitive and dependable diagnostic routine tool for COVID-19 patients along with PCR-based methods.

Recently, a simple, sensitive, and rapid technique was reported by Singh et al. [35] who had reported the detection of the SARS-CoV-2 virus by means of a targeted mass spectrometric protocol from naso-oropharyngeal swabs. The presence of two peptides specific to SARS-CoV-2 virus can be detected employing a multiple reaction monitoring process with $100 \%$ specificity and $95 \%$ sensitivity in comparison to the RT-PCR technique. It was also confirmed that these peptides could be detected even in the patients who have previously recovered from COVID-19 infection having negative test report verified by RT-PCR method. It demonstrates the sensitivity of this novel technique.

In this MS-based detection protocol, the virus can be detected in a time period less than 3 min, while sample preparation time can be carried out in less than $30 \mathrm{~min}$. This novel method can directly detect this virus without even amplifying the RNA during detection, as in the case with RT-PCR. This MS-based technique relies on detection of two peptides which are exclusive to SARS-CoV-2 virus and are not observed in any other coronavirus or other viruses. Though seven peptides were found to be unique to SARSCoV-2 virus, only two peptides such as the spike protein and 
the replicase protein are used for rapid virus detection. The virus in the swab samples were inactivated using surfactant solution and further processed before being used for virus detection. The researchers initially tested the techniques using 14 nasopharyngeal swab samples and then confirmed using 20 controls and 63 samples from COVID-19-positive patients tested by RT-PC method and afterwards recovered fully from infection. Although mass spectrometer equipment is costly, MS-based detection method would charge only about Rs 100 per test $(\sim 1-2)$, and consequently, the technique is cheaper than RT-PCR. It can be used for screening and diagnostic purpose. It can either supplement RT-PCR or be used as an alternative to RT-PCR method with high specificity and sensitivity. The experimental results based on MS-based detection of COVID-19 was published in the Journal of Proteins and Proteomics [35].

\section{Magnetic particle spectroscopy-based detection of COVID-19}

Magnetic particle spectroscopy (MPS), which is also known as magnetization response spectroscopy, is a versatile, highly sensitive inexpensive analytical detection tool for various biological and biomedical assays. It is based on magnetic particle imaging (MPI), which can directly measure the concentration of super magnetic iron oxide nanoparticles. Therefore, MPS can be viewed as a zero-dimensional MPI scanner which can carry out investigation on super magnetic iron oxide nanoparticles spectroscopically.

Professor Jian-Ping Wang and his research team at the University of Minnesota, USA, had developed a transportable diagnostic method based on MPS, which can provide authorization for fast, sensitive detection of SARS-CoV-2 virus [36]. The versatility of MPS technique is the use of artificially designed magnetic nanoparticles for different bioassays where magnetic nanoparticles can act as magnetic tracers as the nanoparticles surfaces were functionalized with test reagents such as antibodies, aptamers, or peptides. COVID-19 antigen can be detected by functionalizing the nanoparticles with polyclonal antibodies to structural proteins nucleocapsid and spike proteins which are the essential components of the coronavirus. The antibodies will permit the magnetic nanoparticles for binding epitopes or receptor sites, on these particular proteins, and MPS technique will monitor and evaluate the real-time specific binding of the nanoparticles with these proteins. The concentration of the target analyte,i.e. protein will signify the presence of Coronavirus and can directly affects the responses of the nanoparticles to the magnetic field. Various magnetic nanosensing methods such as magneto resistance, nuclear magnetic resonance, and magnetic particle spectroscopy can be employed for diagnostics purpose as these can be directly applied for pathogen detection [37, 38]. Therefore, magnetic nanosensors can be utilized to control the recent SARS-CoV-2 outbreak and to prevent the future epidemics.

On the other hand, commonly used PCR approaches are restricted for the detection of nucleic acids only, while detection protocol based on magnetic nanosensors is more versatile and can be applied for the detection of antigens, antibodies, and nucleic acids. Magnetic nanosensor-based diagnostic tools are less popular and frequently unnoticed compared to the traditional optical, electrochemical, and mechanical sensors.

The most common drawback of most magnetic nanosensor-based detection techniques is the use of magnetic labels to detect and separate. Magnetic labels in magnetic nanosensor-based techniques are beneficial over the optical and electrochemical techniques which will use fluorescent dyes. However, unstable enzymes and radioisotopes regarding the biocompatibility and stability in different biological environments and the requirement for a label in the detection process could restrain the application of this versatile technique in many in vivo studies.

\section{FTIR spectroscopy-based detection of COVID-19}

Attenuated total reflectance Fourier transform infrared (ATR-FTIR) spectroscopy is a fast spectroscopic technique, which is dependent on the vibrational signatures of chemical structures and can be employed with a multivariate statistical model for the categorization of various diseases. The use of IR spectroscopy for viral detection is comparatively new [39]. There had been reports on the application of ATRFTIR as a rapid screening test for the detection of COVID19 using plasma [40] or nasopharyngeal swab samples [41].

Zhang et al. [40] demonstrated the potential of ATR-FTIR for testing and principal diagnosis of COVID-19. The spectral differences between COVID-19-infected and healthy controls and the potential spectral markers were detected by multivariate and statistical analysis. The advantage of this technique was a requirement of a very minute volume of serum sample $(\sim 3 \mu \mathrm{L})$ and rapid detection time (few minutes).

On the other hand, Baruana et al. [41] employed ATRFTIR spectroscopy to investigate the saliva samples on pharyngeal swabs procured from individual with or without infection of COVID-19 virus. Their protocol was not intended for substitution of accessible diagnostic methods for COVID-19 like RT-PCR but to provide a fast prescreening tool. Manufactured saliva samples spiked with inactivated $\gamma$-irradiated COVID-19 virus at levels down to 1582 copies/mL can provide IR spectra with high signal-to-noise ratio. Major viral spectral peaks were cautiously related to

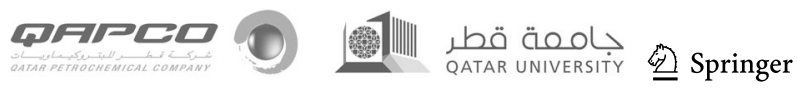


nucleic acids bands, including RNA. The technique can provide a blind sensitivity of $95 \%$ and specificity of $90 \%$.

Recently, Banerjee et al. [25] carried out rapid blood test for diagnosis of COVID-19 disease severity using a group of 160 COVID-19 patients by using ATR-FTIR spectroscopy. A straightforward plasma processing and ATR-FTIR data acquirement process was carried out by them employing $75 \%$ ethanol for viral inactivation. Subsequently, partial least squares-discriminant analysis (PLS-DA) models were developed and tested using data from 130 and 30 patients, respectively. The autonomous test set accomplished 69.2\% specificity and $94.1 \%$ sensitivity. Their study demonstrated the potential of ATR-FTIR spectroscopy as a rapid, costeffective COVID-19 detection tool to assist COVID-19 patient management during an epidemic. The strengths of this study include the rapid sample preparation and ATRFTIR data acquisition methodology and the development of a multivariate algorithm combining ATR-FTIR and clinical data. Although there were 128 samples in the training data set, the independent data set contained only 30 samples. Potential investigations should enhance the sample size to increase the predictive performance.

Kitane et al. [26] had reported an unparalleled, fast, reagent-free, and user-friendly screening spectroscopic method for the detection of SARS-CoV-2 on RNA extracts. This method was verified on clinical samples collected from 280 patients with quantitative predictive scores on both positive and negative samples. The investigation was based on a multivariate analysis of FTIR spectra of RNA extracts. This method was in good agreement with RT-PCR and had accomplished $97.8 \%$ accuracy, $97 \%$ sensitivity, and $98.3 \%$ specificity and the testing time post RNA extraction was reduced from hours to minutes. Additionally, this method can be employed in numerous laboratories with inadequate resources. Figure 2 illustrated the schematic diagram exhibiting sequential FT-IR-based assay for the detection of SARS-CoV-2, which consisted of sample collection, RNA extraction, FTIR analysis, and subsequent machine learning. Compared to RT-PCR method, this method was much faster (1.5 min vs $2-4$ h post-RNA extraction) without using any reagent, and less biohazard waste materials can be produced after completion of the detection test. Even though the method has quite a lot of merits, it might need a considerable amount of samples to augment its sensitivity, specificity, and accuracy. In this work, the researchers had employed machine learning for the first time, to make classification models, which can predict the patient's infection, based on the IR spectra of the extracted RNAs. After a complete literature survey, it can be inferred that this versatile technology based on a dual and balancing amalgamation of FT-IR and machine learning on RNA samples had not been reported so far. From the given high diagnosis performance output obtained, it can be concluded that spectroscopy can serve as a promising tool for viral diagnosis. Experiments were carried out on two sets of samples. The first set of 280 clinical samples was utilized for assessing the sensitivity, specificity, and accuracy, while the second set of synthetic RNA samples was employed to estimate the limit of detection and can further evaluate the selectivity against 15 other respiratory viruses.

\section{Raman spectroscopy-based detection for COVID-19 testing}

Surface-enhanced Raman scattering (SERS) is a popular surface-sensitive spectroscopic analytical technique, which is based on the large enhancement of weak Raman signal and can provide detection of various chemical and biological systems [42-44]. The low sensitivity problem of conventional Raman spectroscopy has been solved as a result of the discovery of SERS.

SERS technique has diverse applications ranging from plasmonics, sensing, and catalysis to biomedical applications and diagnostics [45-49]. This novel powerful analytical technique has been utilized to identify several biomolecules
Fig. 2 The schematic diagram exhibiting sequential FT-IRbased assay for the detection of SARS-CoV-2, which consisted of sample collection, RNA extraction, FTIR analysis, and subsequent machine learning. (Reprinted with permission from Kitane et al. [26]. Copyright (2021) Nature Portfolio)
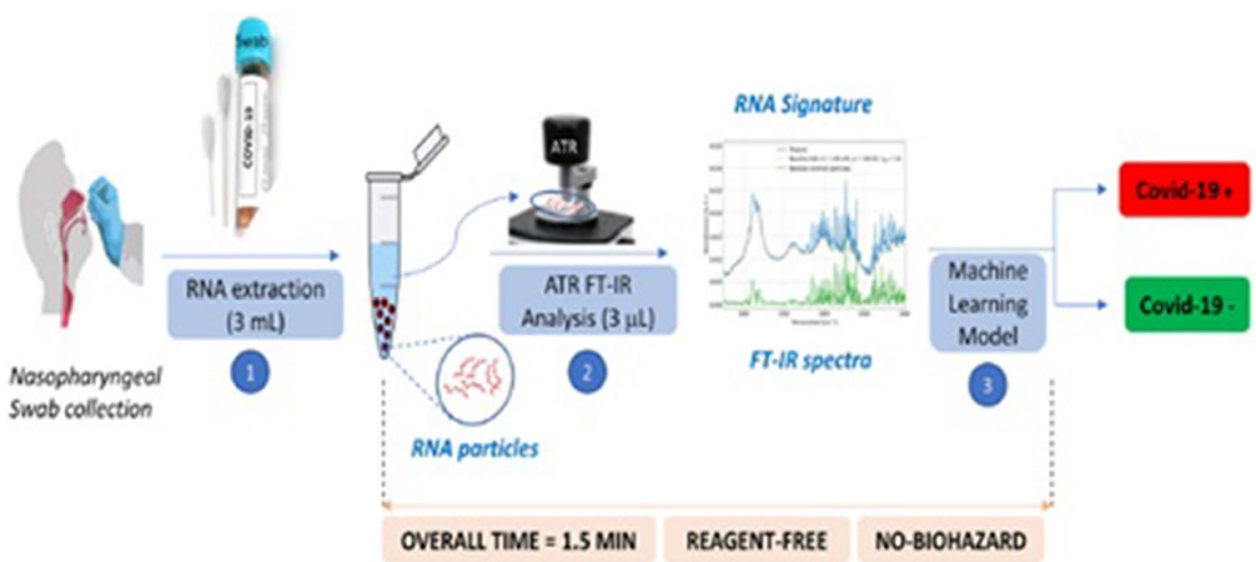
like carbohydrates, proteins, and amino acids both qualitatively and quantitatively [48-53]. SERS can even be used to detect pathogens including bacteria and viruses within a short timescale [48-50].

Professor Miguel Jose Yacaman of Northern Arizona University and his research team had developed an original non-invasive diagnostic protocol utilizing the concepts of nanotechnology, plasmonics, and 2D materials to detect SARS-CoV-2 virus rapidly using single-molecule surface-enhanced Raman spectroscopy (SM-SERS) [54]. The researchers had applied SM-SERS technique to detect spike proteins of the SARS-CoV-2 virus. Yacaman had earlier used SERS to detect glycoproteins and sialic acid during the testing of breast cancer [55]. According to Yacaman, SM-SERS-based diagnostic protocol for SARS-CoV-2 testing could be the first physics-based detection method which would be fast and low-cost, with high sensitivity and specificity, providing a low percentage of incorrect negatives. Portable Raman instrument could permit the test to be used extensively in diverse populations-for instance, in rural communities, or in schools, factories, and community centres, along with conventional testing locations during the development stage.

Scientists in Israel had developed a spectroscopy-based 1-min breath analyzer to detect coronavirus [56]. The device was developed by a team led by Professor Hossam Haick in association with scientists from Wuhan, China, where the novel coronavirus was first detected in December 2019. The breath analyzer was made of nanomaterial-based hybrid sensors that can detect SERS-CoV-2 from precise volatile organic compounds, unique fingerprints for various forms of diseases, in exhaled breath. Results can be obtained under a minute and the test can be performed in meticulous facilities without the need of extensive and expensive laboratory tests. In a controlled clinical study conducted in March 2020 in Wuhan, the breath test device detected coronavirus disease explicit biomarkers in exhaled breath with $92 \%$ accuracy, $100 \%$ sensitivity, and $84 \%$ specificity. The peer-reviewed research was published in the journal ACS Nano [56].

Amit Dutt from the Mumbai-based Tata Memorial Centre and his research team had utilized conventional Raman spectroscopy for the detection of RNA viruses present in saliva samples [57]. It is a proof-of-concept study to analyse noninfectious RNA viruses using conventional Raman spectroscopy without using any additional reagent to enhance the signal. The novel coronavirus was found to be in adequate quantities in human saliva according to their scientific studies. The saliva samples were enhanced with non-infectious RNA viruses and were further analysed it with Raman spectroscopy.

The raw Raman spectroscopy data were analysed and the captured Raman signals were compared with both viral positive and negative samples. Each sample will produce 1400 spectra on which statistical analysis was carried out exhibiting a set of 65 Raman spectral features, which was sufficient enough to detect the viral positive signal. The signal set had $92.5 \%$ sensitivity and $88.8 \%$ specificity. The results was published in the Journal of Biophotonics [57].

To curtail inconsistency and systematize the analysis of the Raman spectra for RNA viruses, they developed an automated tool, which was RNA virus detector using a graphical user interface. The automated tool was used for identifying the RNA virus from an individual or a group of samples both explicitly with high reproducibility. This tool can collect raw data from a Raman spectroscopy-based analysis carried out on the 65-spectra signature and will provide an objective output if viral RNA is present or absent in the sample. This concept of detection of RNA viruses from saliva sample could generate the foundation for field application of Raman spectroscopy to handle viral epidemics, like the ongoing COVID-19 pandemic. This Raman spectroscopy-based protocol can be used only for monitoring purpose, as this novel detection tool can only recognize RNA viruses such as common cold virus or HIV and not detect the specific one. It cannot detect COVID-19 viral-specific signature. The major benefit is that the detecting device can be engaged to the field and people who test positive for RNA virus can be quarantined while another sample may be sent for validation using RT-PCR. The technique is rapid as the entire procedure of data procurement and analysis can be completed within a minute. Subsequently, no extra reagent is required, and there is no recurrent charge. By installing portable Raman spectrophotometer at the entry point such as airport, railway station or shopping mall or entrance of campus of big housing complex, office, school, college, university, or research institutes, one can rapidly monitor people within minutes.

Carlomango et al. [58] had introduced a Raman spectroscopy-based detection protocol by analysing salivary samples from COVID-19-infected patients. Their Raman fingerprint data had provided strong evidence of infection by presenting biochemical signature of the saliva samples of patients affected by COVID-19 and a novel, non-invasive, and rapid detection technique was introduced with high accuracy, precision, specificity, and sensitivity more than $90 \%$.

Sanchez et al. [27] had recently detected SARS-CoV-2 and its $\mathrm{S}$ and $\mathrm{N}$ proteins using SERS technique. They demonstrated for the first time that the spectroscopic signature of the SARS-CoV-2 virus can be obtained and the spectra were primarily associated with the signals from the $S$ and $\mathrm{N}$ proteins. They suggested that rapid, cost-effective, and trustworthy clinical test using SERS can be further developed. They fabricated gold nanostar nanoparticles, which was employed as SERS-active substrate by combining with $\mathrm{MoS}_{2}$ thin layers and a very reliable signature for SARS$\mathrm{CoV}-2$ virion particles was monitored. Additionally, the

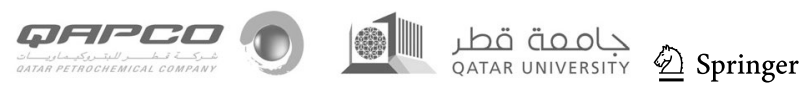


main proteins, $\mathrm{S}$ and $\mathrm{N}$, will generate well-defined spectra, which suggests that SERS spectroscopy can be used not only for virus detection but also to investigate the structure of the proteins and track the in situ transformation formed by various conditions.

In general, the spectra of the SARS-CoV-2 virus and the $\mathrm{S}$ and $\mathrm{N}$ proteins demonstrated broad peaks, which are the result of many overlapping smaller peaks related to several molecular vibrations. To obtain an accurate analysis of the virus and proteins, it is essential to deconvolute the peaks into individual components. This can be demonstrated by simulation of the Raman-SERS spectra. Vibrational frequency simulations were calculated using the Gaussian code based on the Hartree-Fock approximation for an unrestricted isolated molecule, which is part of the $\mathrm{N}$ protein to obtain the Raman spectra. The $\mathrm{N}$ and $\mathrm{S}$ proteins contain a very large number of diverse molecules and, thus, its full structure will be very difficult to simulate.

As the study carried out by them was at the early stage, clinical testing and direct comparison to other tests was essential to evaluate the sensitivity and specificity of SERS. Nevertheless, their study exhibits immense potential for further development of this technique. It can be concluded that if the technique can be further developed, SERS test on saliva sample will make instantaneous outcomes and use of nanoparticles in the form of SERS active substrate is essential which can be especially synthesized at low cost. Figure 3 shows the SERS spectra of (a) the N protein, (b) the S protein, and (c) the inactivated SARS-CoV-2 virion particles, while Fig. 4 shows the theoretical density functional theory

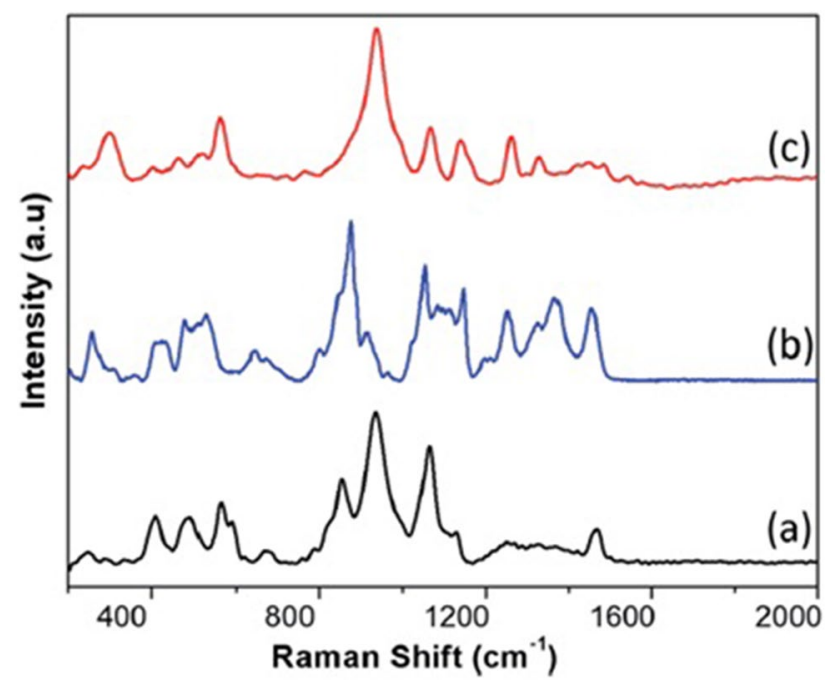

Fig. 3 The SERS spectra of (a) the N protein, (b) the $\mathrm{S}$ protein, and (c) the inactivated SARS-CoV-2 virion particles. (Reprinted with permission from Sanchez et al. [27]. Copyright (2021) Royal Society of Chemistry)

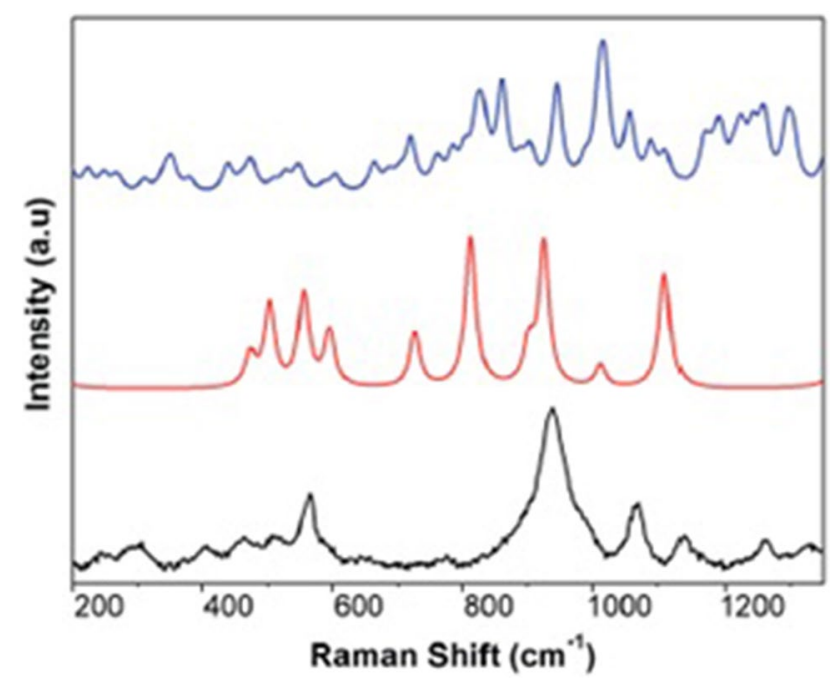

Fig. 4 The theoretical DFT simulation of portions of the Raman spectra of components of the protein N. (Reprinted with permission from Sanchez et al. [27]. Copyright (2021) Royal Society of Chemistry)

simulation of portions of the Raman spectra of components of the protein $\mathrm{N}$.

Zhang et al. [28] had developed an assay using SERS combined with multivariate analysis to identify SARSCoV-2 in an ultrarapid way without any further pretreatment such as RNA extraction. Silver nanorod employed as SERS array was functionalized with cellular receptor ACE2, and strong SERS signals of ACE2 at 1032, 1051, 1089, 1189, 1447 , and $1527 \mathrm{~cm}^{-1}$ were monitored. The recognition and binding of receptor binding domain of SARS-CoV-2 spike protein on SERS assay significantly quenched the spectral intensities of most peaks and exhibited a shift from 1189 to $1182 \mathrm{~cm}^{-1}$. On-the-spot tests on 23 water samples were carried out employing a portable Raman spectrometer with high accuracy and efficiency for spot detection of SARS-CoV-2. Various other studies such as evaluation of disinfection performance, exploration of viral survival in environmental media, evaluation of viral decay in wastewater treatment plant, and tracking of SARS-CoV-2 in pipe network were also carried out by them. Presently, the fabricated SERS assay had satisfactory accuracy with false-positive and false-negative percentages, which can be further enhanced by fabrication accomplishment, database system, and algorithm upgradation. It has a brilliant prospect and enormous potential as a fast and on-site detection tool for SARS-CoV-2 and other viruses. Figure 5 shows the SERS spectra of ACE2 proteins and 23 tested water samples by the SERS assay (mean value) using a portable Raman spectrometer.

New diseases can emerge or re-emerge at any time and cause epidemics. Diseases associated with the novel coronavirus have been underrated so far, and since the SARS 
Fig. 5 The SERS spectra of ACE2 proteins and 23 tested water samples by the SERS assay (mean value) using a portable Raman spectrometer. (Reprinted with permission from Zhang et al. [28]. Copyright (2021) Elsevier Inc.)

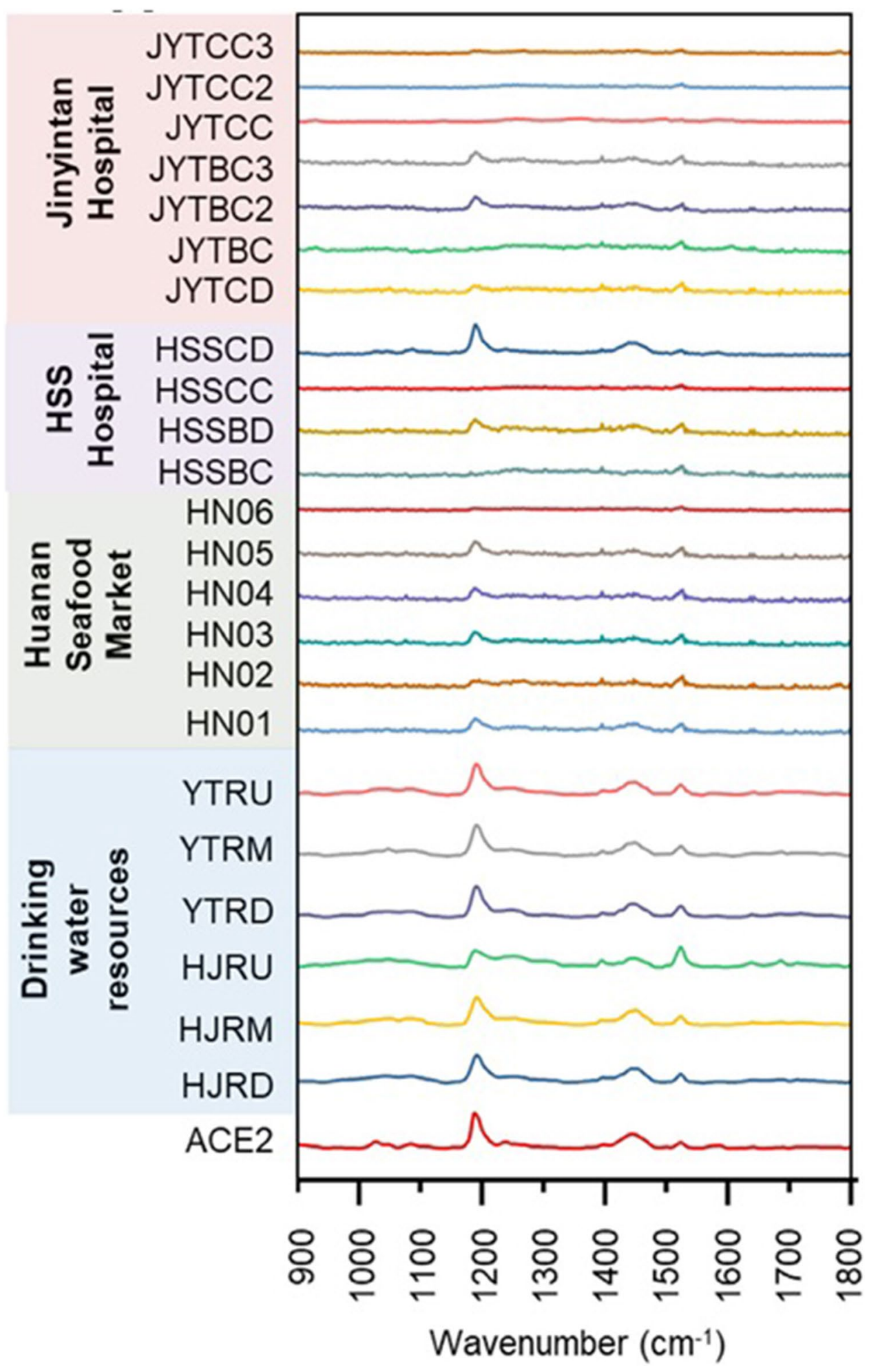

COVID-19 pandemic will be valuable for the next pandemic to come. Therefore, judicious rapid detection along with the medical authentication of sensitivity and specificity of diagnostic technique is important to intimidate the diagnostic challenges. Spectroscopy can provide the capable method and MERS epidemics were not utilized efficiently to progress medical infrastructure to rapidly activate detection tests, development of vaccines, antiviral drugs, and policies to regulate viral epidemic and to organize health care systems all over the world. The lessons from the current

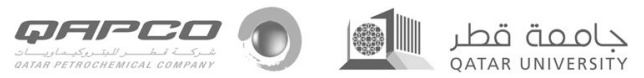
QATAR UNIVERSITY 
through the potential disease molecular/chemical biomarkers. IR or/and Raman spectroscopy-based devices for the detection of viruses and bacteria can be further developed through the analysis of the structure of proteins and nucleic acids in the virus. Spectroscopic methods can be significant to provide quick, precise, and comparatively inexpensive protocols of detecting viral and bacterial infections and observing their structural change with the surrounding environment. The experimental observation is even more vital as viruses are known to change their RNA structure with respect to the surrounding environment making it more difficult to combat the viral infections. This change in RNA structure is normally recognized as mutation in biological terminology and further investigation of mutation process is vital for the development of vaccines and to fight against this deadly viral infection. It is expected that one can develop novel detection system as investigative tool to study COVID19 samples by employing IR and Raman spectroscopies.

\section{Conclusions}

Over the past 100 years, the advent of various viruses, which can cause a widespread variation of diseases both in human and in other animals, had happened. It is expected that these viruses will remain to appear and cause human epidemics owing to their capability to recombine, mutate, and infect multiple species and cell types. Forthcoming research on viruses will continue to explore many characteristics of viral replication and pathogenesis. Lately, the outbreak of COVID-19 has huge effect worldwide, and premature rapid uncovering of SARS-CoV-2 is crucial for battling this deadly virus. Spectroscopy can provide early detection, diagnosis, and monitoring of several human diseases. Both IR and Raman spectroscopy can be applied for the early and rapid detection of pathogens. Spectroscopic techniques have several advantages than the conventional RT-PCR method for detection of this virus. Spectroscopic methods are novel as well as versatile as they can provide rapid, precise, and comparatively inexpensive reagent-free protocols of detecting viral and bacterial infections and observing their structural change with the surrounding environment. Spectroscopic techniques can be ideal as well as suitable and it is expected that spectroscopy will provide versatile diagnostic tool in the development of a rapid non-invasive diagnostic system for detecting the novel coronavirus without using any reagent. Application of various spectroscopic techniques for the fast, delicate, explicit, and economical detection of COVID-19 has been illustrated in these review articles. Application of various spectroscopic techniques is in the early development stage. It is expected that spectroscopic techniques will replace conventional detection methods such as RT-PCR and RAT in the near future and will make a breakthrough for the complete rapid diagnosis of COVID-19. We hope that proper inexpensive method and rapid diagnosis of this new virus will help the people throughout the globe to start normal functioning as usual and enable the development of proper vaccines and antiviral medicines to combat SERS-CoV-2 like virus epidemic in the coming years and in the future. We congratulate all the unsung heroes who worked so far to help the world combat this pandemic.

Acknowledgements U. K. S. would like to thank Indian National Science Academy (INSA), New Delhi, India, for INSA Visiting Scientist Fellowship (SP/VF-9/2014-15/273/01 April, 2014) at Bio-Inspired Materials Research Laboratory, Department of Chemistry, Savitribai Phule Pune University, Ganeshkhind, Pune-411007, India). U. K. S. would like to acknowledge financial support from the projects funded by the DHESTBT, Government of West Bengal (memo no. 161(sanc)/ ST/P/S\&T/9G-50/2017 dated 8/2/2018.

Funding This study was funded by DHESTBT (Department of Higher Education, Science, Technology and Biotechnology), Government of West Bengal (Memo no. 161(sanc)/ST/P/S\&T/9G-50/2017 dated $8 / 2 / 2018$ )

\section{Declarations}

Conflict of interest The authors declare no competing interests.

\section{References}

1. A.R. Fehr, S. Perlman, Coronaviruses: an overview of their replication and pathogenesis. Methods Mol Bio 1282, 1-23 (2015)

2. P. Sun, X. Lu, C. Xu, W. Sun, B. Pan, Understanding of COVID19 based on current Evidence. J Med Virol 92, 548-551 (2020)

3. C.S. Goldsmith, K.M. Tatti, T.G. Ksiazek, P.E. Rollin, J.A. Comer, W.W. Lee et al., Ultrastructural characterization of SARS coronavirus. Emerg Infect Dis 10, 320-326 (2004)

4. Details on COVID-19; Public Health Image Library (PHIL), Centers for Disease Control and Prevention. https://phil.cdc.gov/Detai 1s.aspx?pid=23354 (accessed 2020/03/27)

5. H.Y. Yang, G.C. Duan, Analysis on the epidemic factors for the Corona Virus Disease. Zhonghua Yu Fang Yi Xue Za Zhi 54, 608-613 (2020)

6. X. Yang, Y. Yu, J. Xu, H. Shu, J. Xia, H. Liu, Y. Wu, L. Zhang, Z. Yu, M. Fang, T. Yu, Y. Wang, S. Pan, X. Zou, S. Yuan, Y. Shang, Clinical course and outcomes of critically ill patients with SARSCoV-2 pneumonia in Wuhan, China: a single-centered, retrospective, observational study. Lancet Respir Med 8, 475-481 (2020)

7. H. Lu, C.W. Stratton, Y.-W. Tang, Outbreak of Pneumonia of Unknown Etiology in Wuhan, China: the mystery and the miracle. J Med Virol 92, 401-402 (2020)

8. N. Zhu, D. Zhang, W. Wang, X. Li, B. Yang, J. Song, X. Zhao, B. Huang, W. Shi, R. Lu, A Novel coronavirus from patients with pneumonia in China, 2019. N Engl J Med 382, 727-733 (2020)

9. F. Wu, S. Zhao, B. Yu et al., A new coronavirus associated with human respiratory disease in China. Nature 579, 265-269 (2020)

10. J.P. Harrison, D. Berry, Vibrational spectroscopy for imaging single microbial cells in complex biological samples. Front Microbiol 8, 1-7 (2017)

11. A.C.S. Talari, Z. Movasaghi, S. Rehman et al., Raman spectroscopy of biological tissues. Appl Spectrosc Rev 50, 46-111 (2015)

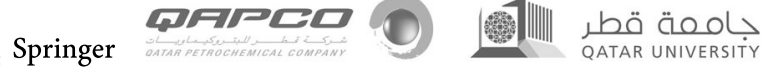


12. H. Barr, O. Old, M. Almond et al., Vibrational spectroscopy: the solution for immediate medical diagnosis. Mater Today Proc 2, 890-893 (2015)

13. C. Krafft, J. Popp, The many facets of Raman spectroscopy for biomedical analysis. Anal Bioanal Chem 407, 699-717 (2015)

14. E.J. Saude, C.M. Slupsky, B.D. Sykes, Optimization of NMR analysis of biological fluids for quantitative accuracy. Metabolomics 2, 113-123 (2006)

15. R.S. Khan, I.U. Rehman, Spectroscopy as a tool for detection and monitoring of Coronavirus (COVID-19). Expert Rev Mol Diagn 20, 647-649 (2020)

16. Z. Movasaghi, S. Rehman, I.U. Rehman, Fourier transform infrared (FTIR) spectroscopy of biological tissues. Appl Spectrosc Rev 43, 134-179 (2008)

17. S.Y. Lin, M.J. Li, W.T. Cheng, FT-IR and Raman vibrational micro-spectroscopies used for spectral bio-diagnosis of human tissues. Spectrosc 21, 1-30 (2007)

18. A.E. Gorbalenya et al., The species severe acute respiratory syndrome-related coronavirus: classifying 2019-NCoV and naming it SARS-CoV-2. Nat Microbiol 5, 536-544 (2020)

19. P. Zhou, X.-L. Yang, X.-G. Wang et al., A pneumonia outbreak associated with a new coronavirus of probable bat origin. Nature 579, 270-273 (2020)

20. B. Udugama, P. Kadhiresan, H.N. Kozlowski, A. Malekjahani, M. Osborne, V.Y.C. Li, H. Chen, S. Mubareka, J.B. Gubbay, W.C.W. Chan, Diagnosing Covid-19: the disease and tools for detection. ACS Nano 14, 3822-3835 (2020)

21. W.C.W. Chan, Nano research for covid-19. ACS Nano 14, 3719$3720(2020)$

22. I. Schroder, COVID-19: a risk assessment perspective. ACS Chem Health Saf 27, 160-169 (2020)

23. I.A. Mattioli, A. Hassan, O.N. Oliveira Jr., F.N. Crespilho, on the challenges for the diagnosis of SARS-CoV-2 based on a review of current methodologies. ACS Sens 5, 3655-3677 (2020)

24. R. Medhi, P. Srinoi, N. Ngo, H.-V. Tran, T.R. Lee, Nanoparticlebased strategies to combat COVID-19. ACS Appl Nano Mater 3, 8557-8580 (2020)

25. A. Banerjee, A. Gokhale, R. Bankar, V. Palanivel, A. Salkar, H. Robinson, J.S. Shastri, S. Agrawal, G. Hartel, M.M. Hill, S. Srivastava, Rapid classification of COVID-19 severity by ATR-FTIR spectroscopy of plasma samples. Anal Chem 93, 10391-10396 (2021)

26. D.L. Kitane, S. Loukman, N. Marchoudi, A. Fernandez-Galiana, F.Z. El Ansari, F. Jouali, J. Badir, J.L. Gala, D. Bertsimas, N. Azami, O. Lakbita, O. Moudam, R. Benhida, J. Fekkak, A simple and fast Spectroscopy-based technique for Covid-19 diagnosis. Sci Rep 11, 16740 (2021)

27. J.E. Sanchez, S.A. Jaramillo, E. Settles, J.J. Velazquez Salazar, A. Lehr, J. Gonzalez, C. Rodri1guez Aranda, H.R. Navarro-Contreras, M.O. Raniere, M. Harvey, D.M. Wagner, A. Koppisch, R. Kellar, P. Keimb, M.J. Yacaman, Detection of SARS-CoV-2 and its $\mathrm{S}$ and $\mathrm{N}$ proteins using surface enhanced Raman spectroscopy. RSC Adv 11, 25788-25794 (2021)

28. D. Zhang, X. Zhang, R. Ma, S. Deng, X. Wang, X. Wang, X. Zhang, X. Huang, Y. Liu, G. Li, J. Qu, Y. Zhu, J. Li, Ultra-fast and onsite interrogation of severe acute respiratory syndrome coronavirus 2 (SARS-CoV-2) in waters via surface enhanced Raman scattering (SERS). Water Res. 200, 117243-117251 (2021)

29. R.A. Khailany, M. Safdar, M. Ozaslan, Genomic characterization of a novel SARS-CoV-2. Gene Rep 19, 100682 (2020)

30. L.J. Carter, L.V. Garner, J.W. Smoot, Y. Li, Q. Zhou, C.J. Saveson, J.M. Sasso, A.C. Gregg, D.J. Soares, T.R. Beskid, S.R. Jervey, C. Liu, Assay techniques and test development for COVID-19 diagnosis. ACS Cent Sci 6, 591-605 (2020)
31. A. Tahamtan, A. Ardebili, Real-time RT-PCR in COVID-19 detection: issues affecting the results. Expert Rev Mol Diagn 20, 453-454 (2020)

32. V.M. Corman, O. Landt, M. Kaiser et al., Detection of 2019 novel coronavirus (2019-nCoV) by real-time RT-PCR. Euro Surveill 25, 2000045 (2020)

33. M. Shen, Y.Y. Zhou, J. Ye, A.A. Abdullah Al-Maskri, Y. Kang, S. Zeng, S. Cai, Recent advances and perspectives of nucleic acid detection for coronavirus. J Pharm Anal 10, 97-101 (2020)

34. C. Ihling, D. Tänzler, S. Hagemann, A. Kehlen, S. Hüttelmaier, C. Arlt, A. Sinz, Mass spectrometric identification of SARSCoV-2 proteins from gargle solution samples of COVID-19 patients. J Proteome Res 19, 4389-4392 (2020)

35. P. Singh, R. Chakraborty, R. Marwal, V.S. Radhakrishan et al., A rapid and sensitive method to detect SARS-CoV-2 virus using targeted-mass spectrometry. J Protein Proteomics 11, 159-165 (2020)

36. K. Wu, R. Saha, D. Su, V.D. Krishna, J. Liu, M.C.-J. Cheeran, J.-P. Wang, Magnetic-nanosensor-based virus and pathogen detection strategies before and during COVID-19. ACS Appl Nano Mater 3, 9560-9580 (2020)

37. D. Su, K. Wu, V. Krishna, T. Klein, J. Liu, Y. Feng, A.M. Perez, M.C. Cheeran, J.-P. Wang, Detection of influenza A virus in swine nasal swab samples with a wash-free magnetic bioassay and a handheld giant magneto resistance sensing system. Front. Microbiol 10, 1-10 (2019)

38. T. Aytur, J. Foley, M. Anwar, B. Boser, E. Harris, P.R. Beatty, A novel magnetic bead bioassay platform using a microchip-based sensor for infectious disease diagnosis. J Immunol Methods 314, 21-29 (2006)

39. J.N. Fernandes, L.M.B. Dos Santos, T. Chouin-Carneiro, M.G. Pavan, G.A. Garcia, M.R. David, J.C. Beier, F.E. Dowell, R. Maciel-de-Freitas, M.T. Sikulu-Lord, Rapid, noninvasive detection of Zika virus in Aedes aegypti mosquitoes by near-infrared spectroscopy. Sci Adv 4, 1-6 (2018)

40. L. Zhang, M. Xiao, Y. Wang, S. Peng, Y. Chen, D. Zhang, D. Zhang, Y. Guo, X. Wang, H. Luo, Q. Zhou, Y. Xu, Fast screening and primary diagnosis of Covid-19 by ATR-FTIR-IR. Anal Chem 93, 2191-2199 (2021)

41. V. G. Barauna, M. N. Singh, L. L. Barbosa, W. D. Marcarini, P. F. Vassallo, J. M. Mill, R. Ribeiro-Rodrigues, L. C. G. Campos, P. H. Warnke, F. L. Martin, Anal. Chem. Ultrarapid on-site detection of SARS-CoV-2 infection using simple ATR-FTIR spectroscopy and an analysis algorithm: high sensitivity and specificity. 93, 2950-2958 (2021)

42. U.K. Sur, Surface-enhanced raman spectroscopy: recent advancement of Raman spectroscopy. Resonance 15, 154-164 (2010)

43. U.K. Sur, J. Chowdhury, Surface-enhanced Raman scattering: overview of a versatile technique used in electrochemistry and nanoscience. Curr. Sci. 105, 923-939 (2013)

44. U.K. Sur, Surface-enhanced Raman scattering (SERS) spectroscopy: a versatile spectroscopic and analytical technique used in nanoscience and nanotechnology. Adv Nano Res 1, 111-124 (2013)

45. K. Gracie, E. Correa, S. Mabbott, J.A. Dougan, D. Graham, R. Goodacre, K. Faulds, Simultaneous detection and quantification of three bacterial meningitis pathogens by SERS. Chem. Sci. 5, 1030-1040 (2014)

46. K. F. Domke, Surface enhanced Raman spectroscopy, analytical, biophysical and life science applications. Edited by Sebastian Schlücker, Angew. Chem. Int. Ed. 50, 8226-8226 (2011)

47. A. Wang, Y.F. Huang, U.K. Sur, D.Y. Wu, B. Ren, S. Rondinini, C. Amatore, Z.Q. Tian, In situ identification of intermediates of benzylchloride reduction at a silver electrode by SERS coupled with DFT calculations. J Am Chem Soc 13, 9534-9536 (2010) 
48. B. Ankamwar, U.K. Sur, P. Das, SERS study of bacteria using biosynthesized silver nanoparticles as SERS substrate. Anal Methods 8, 2335-2340 (2016)

49. U.K. Sur, B. Ankamwar, S. Karmakar, A. Halder, P. Das, Green synthesis of silver nanoparticles using the plant extract of Shikakai and Reetha. Mater Today Proc 5, 2321-2329 (2018)

50. T.T. Liu, Y.H. Lin, C.S. Hung, T.J. Liu, Y. Chen, Y.C. Huang, T.H. Tsai, H.H. Wang, D.W. Wang, J.K. Wang, Y.L. Wang, C.H. Lin, A High Speed Detection Platform based on surface-enhanced Raman scattering for monitoring antibiotic-induced chemical changes in bacteria cell wall. PLoS ONE 4, 1-10 (2009)

51. C.-H. Chuang, Y.-T. Chen, Raman scattering of L-tryptophan enhanced by surface plasmon of silver nanoparticles: vibrational assignment and structural determination. J Raman Spectrosc 40, 150-156 (2009)

52. R.M. Jarvis, R. Goodacre, Discrimination of bacteria using surface-enhanced Raman spectroscopy. Anal Chem 76, 40-47 (2004)

53. A. Krolikowska, J. Bukowska, Surface-enhanced resonance Raman spectroscopic characterization of cytochrome c immobilized on 2-mercaptoethanesulfonate monolayers on silver. J Raman Spectrosc 41, 1621-1631 (2010)

54. M.J. Yacaman, Rapid development of a new test for SARS-CoV-2 using single molecule surface enhanced raman spectroscopy (National Science Foundation, USA, 2020)
55. A.H. Arteaga, J. Zermeo Nava, E.S. Machuca, J.J. Salazar, F. Vinogradova, M.J. Yacaman, H.R. Navarro-Contreras, Diagnosis of breast cancer by analysis of sialic acid and concentrations in human saliva by surface enhanced Raman spectroscopy of silver nanoparticles. Nano Res 10, 3662-3670 (2017)

56. B. Shan, Y.Y. Broza, W. Li, Y. Wang, S. Wu, Z. Liu, J. Wang, S. Gui, L. Wang, Z. Zhang, W. Liu, S. Zhou, W. Jin, Q. Zhang, D. Hu, L. Lin, Q. Zhang, W. Li, J. Wang, H. Liu, Y. Pan, H. Haick, Multiplexed nanomaterial-based sensor array for detection of COVID-19 in exhaled breath. ACS Nano 14, 12125-12132 (2020)

57. S. Desai, S.V. Mishra, A. Joshi, D. Sarkar, A. Hole, R. Mishra, S. Dutt, M.K. Chilakapati, S. Gupta, A. Dutt, Raman spectroscopy based detection of RNA viruses in saliva: a preliminary report. $\mathrm{J}$ Biophotonics 13, e202000189 (2020)

58. C. Carlomagno, D. Bertazioli, A. Gualerzi, S. Picciolini, P.I. Banfi, A. Lax, E. Messina, J. Navarro, L. Bianchi, A. Caronni, F. Marenco, S. Monteleone, C. Arienti, M. Bedoni, COVID-19 salivary Raman fingerprint: innovative approach for the detection of current and past SARS-CoV-2 infections. Sci. Rep. 11, 4943 (2021) 